\title{
Capacity Analysis of Correlated Multiple Antenna Systems With Finite Rate Feedback
}

\author{
Jun Zheng and Bhaskar D. Rao \\ University of California at San Diego \\ La Jolla, CA 92093-0403 \\ Email: juzheng@ucsd.edu, brao@ece.ucsd.edu
}

\begin{abstract}
We consider in this paper the analysis of transmit beamforming methods in multiple antenna systems over correlated fading channels and with finite rate feedback of the channel state information. The problem is formulated as a general vector quantization problem with encoder side information, constrained quantization space and non-mean-square distortion function. By utilizing the high-resolution distortion analysis of the generalized quantizer, which is applicable to a wide range of scenarios, we obtain a tight lower bound on the capacity loss of the finite rate quantized MISO system over correlated fading channels. The lower bound of the capacity loss of correlated MISO channels is a generalization of existing results available for i.i.d. channels. The bound, in addition to providing insight into the exact nature of dependence of the quantization loss on the channel correlation matrix, indicates that the loss is less than that of the i.i.d. channels but with the same exponential decaying factor w.r.t. the feedback rate. The generality of the framework is further demonstrated by considering its application to the analysis of suboptimal mismatched channel quantizers, i.e. quantizers designed with an incorrect channel covariance matrix, and comparing it to systems with optimal quantizers. Finally, numerical and simulation results of the finite rate quantized MISO beamforming system with codebook designed by the Lloyd algorithm are presented that confirm the accuracy of the obtained analytical results.
\end{abstract}

\section{INTRODUCTION}

Communication systems using multiple antennas at both the transmitter and the receiver have recently received much attention due to their promise of providing significant capacity increases in a wireless fading environment. The performance of the multiple antenna systems depends heavily on the availability of the channel state information (CSI) at the transmitter (CSIT) and at the receiver (CSIR). Most of the MIMO system design and analysis adopt one of two extreme CSIT assumptions, complete CSIT and no CSIT. In this paper, we consider systems with CSI assumptions in between these extremes. We assume perfect CSIR is available at the receiver, and focus our attention on MIMO systems where CSI is conveyed from the receiver to the transmitter through a finite rate feedback link. Recently, several interesting papers have appeared, proposing design algorithms as well as analytically quantifying the performance of the finite rate feedback multiple antenna systems [1] - [8]. This paper attempts to add to this body of knowledge.

Narula et. al. considered in [1] a multiple transmit antennas and single receive antenna (MISO) system which employs finite-rate feedback to describe the beamforming vector. The Lloyd algorithm [2] was utilized to designing the optimum beamforming vector codebook, where both the channel gain

This research was supported in part by CoRe grant No.02-10109 sponsored by Ericsson and in part by the U. S. Army Research Office under the MultiUniversity Research Initiative (MURI) grant \# W911NF-04-1-0224. and the system mutual information were used as performance metrics. Based on the geometrical properties of the channel space, Mukkavilli et. al. [3] derived a universal lower bound on the outage probability of quantized MISO beamforming systems with arbitrary number of transmit antennas $t$ over i.i.d. Rayleigh fading channels. Love et. al. [4] proposed a codebook design criterion based on minimizing the maximum inner product of the beamforming vectors in the codebook, and related the min-max problem to that of Grassmannian line packing which is the problem of maximally separating lines in the Grassmann manifold. The authors also investigated in [5] the problem of quantizing the beamforming vector under a per-antenna power constraint, which is named as quantized equal gain transmission. This problem was recently revisited by Murthy et. al. in [6] and a closed form capacity loss analysis was obtained.

Vector quantization (VQ) techniques combined with Lloyd algorithm was utilized by Xia et. al. in [7] and Roh et. al. in [8]. The authors derived an (weighted) inner product criterion and used the Lloyd algorithm [2] to generate the codebook that specifically optimize for both the statistical distribution of the vector (or matrix) channel as well as the specific performance metric (for example, the mutual information rate). Both of these groups analyzed the performance of MISO systems with limited-rate feedback in the case of i.i.d. Rayleigh fading channels, and obtained closed form expressions of the capacity loss (or SNR loss) in terms of feedback rate $B$ and antenna size $t$.

In this paper, we consider the analysis of transmit beamforming methods in multiple antenna systems over correlated fading channels and with finite rate CSI feedback. The problem is formulated as a general vector quantization problem with encoder side information, constrained quantization space and non-mean-square distortion function. By utilizing the highresolution distortion analysis of the generalized quantizer provided in [9], which is applicable to a wide range of scenarios, we obtain a tight lower bound on the capacity loss of the finite rate quantized MISO systems over correlated fading channels. The lower bound of the capacity loss of correlated MISO channels are a generalization of existing results available on i.i.d. channels, and its approximations in high-SNR regimes are also provided. The bound provides insight into the exact nature of dependence of the quantization loss on the channel correlation matrix, which indicates that the loss is less than that of the i.i.d. channels but with the same exponential decaying factor w.r.t. the feedback rate. The generality of the framework is further demonstrated by considering its application to the analysis of suboptimal mismatched channel quantizers, i.e. quantizers designed with an incorrect channel covariance 
matrix, and comparing it to systems with optimal quantizers. Finally, numerical and simulation results of the finite rate quantized MISO beamforming system with codebook designed by the Lloyd algorithm are presented that confirm the accuracy of the obtained analytical results.

\section{System ModeL}

We consider an MISO system with $t$ transmit antennas, one single receive antenna, signaling through a frequency flat fading channel. The block fading channel model can be represented as

$$
y=\mathbf{h}^{H} \mathbf{x}+n,
$$

where $y$ is the received signal (scalar), $n \sim \mathcal{N}_{\mathrm{c}}(0,1)$ is the additive complex Gaussian noise with zero mean and unit variance, and $\mathbf{h}^{H} \in \mathbb{C}^{1 \times t}$ is the MISO channel response with distribution given by $\mathbf{h} \sim \mathcal{N}_{\mathrm{c}}\left(\mathbf{0}, \boldsymbol{\Sigma}_{\mathrm{h}}\right)$. For the sake of fair comparison, we normalize the channel covariance matrix such that the mean of the eigen values equals to one, which is the same as in the case of i.i.d. fading channel with $\boldsymbol{\Sigma}_{\mathrm{h}}=I_{t}$. The transmitted signal vector is normalized to have a power constraint given by $E\left[\|\mathbf{x}\|^{2}\right]=\rho$, with $\rho$ representing the average signal to noise ratio at each receive antenna.

It is assumed that there exists a finite rate feedback link of $B$ $\left(N=2^{B}\right)$ bits per channel update between the transmitter and receiver. To be specific, a codebook $\mathcal{C}=\left\{\widehat{\mathbf{v}}_{1}, \cdots, \widehat{\mathbf{v}}_{N}\right\}$, which is composed of unit norm transmit beamforming vectors, is assumed known to both the receiver and the transmitter. Based on the channel realization $\mathbf{h}$, the receiver selects the best code point $\widehat{v}$ from the codebook and sends the corresponding index back to the transmitter. At the transmitter, the unit norm vector $\widehat{\mathbf{v}}$ is employed as the beamforming vector, i.e.

$$
y=\mathbf{h}^{H} \cdot(\widehat{\mathbf{v}} \cdot s)+n=\|\mathbf{h}\| \cdot\langle\mathbf{v}, \widehat{\mathbf{v}}\rangle \cdot s+n, \quad E\left[|s|^{2}\right]=\rho,
$$

where $\mathbf{v}$ is the channel directional vector given by $\mathbf{v}=\mathbf{h} /\|\mathbf{h}\|$. The corresponding ergodic capacity or the maximum system mutual information rate of the quantized MISO beamforming system is given by

$$
C_{\mathrm{Q}}=E_{\mathbf{h}}\left[\log _{2}\left(1+\rho \cdot\|\mathbf{h}\|^{2} \cdot|\langle\mathbf{v}, \widehat{\mathbf{v}}\rangle|^{2}\right)\right] .
$$

With perfect channel state information available at the transmitter $(B=\infty)$, it is optimal to choose $\mathbf{v}=\mathbf{h} /\|\mathbf{h}\|$ as the beamforming vector, and the corresponding system ergodic capacity is given by

$$
C_{\mathrm{p}}=E_{\mathbf{h}}\left[\log _{2}\left(1+\rho \cdot\|\mathbf{h}\|^{2}\right)\right] .
$$

Therefore, the system capacity loss due to the finite rate quantization of the transmit beamforming vectors can be represented as

$C_{\mathrm{L}}=C_{\mathrm{p}}-C_{\mathrm{Q}}=E\left[-\log _{2}\left(1-\frac{\rho \alpha}{1+\rho \alpha} \cdot\left(1-|\langle\mathbf{v}, \widehat{\mathbf{v}}\rangle|^{2}\right)\right)\right]$,

where $\alpha=\|\mathbf{h}\|^{2}$ is the norm square (or the power gain) of the vector channel response.

\section{General Vector Quantization Problem}

The multiple antenna systems with finite-rate feedback can be modeled as a generalized vector quantization problem with additional attributes such as encoder side information, constrained quantization space and non mean-squared distortion measures. High resolution tools commonly used in classical vector quantization have been extended to deal with this generalized problem [9]. We briefly summarize in this section the high-resolution analysis results of the generalized vector quantizer, which are then specialized to provide a tight lower bound on the capacity loss of the finite rate quantized MISO systems over correlated fading channels.

It is first assumed that the source variable $\mathbf{x}=(\mathbf{y}, \mathbf{z})$ is a two-vector tuple with vector $\mathbf{y} \in \mathbb{Q}$ representing the actual quantization variable of dimension $k_{\mathrm{q}}$ and $\mathbf{z} \in \mathbb{Z}$ being the additional side information of dimension $k_{\mathrm{z}}$. The encoder side information $\mathbf{z}$ is available at the encoder but not at the decoder. Based on a particular source realization $\mathbf{x}$, the encoder (or the quantizer) represents vector $\mathbf{y}$ by one of the $N$ vectors $\widehat{\mathbf{y}}_{1}, \widehat{\mathbf{y}}_{2}, \cdots, \widehat{\mathbf{y}}_{N}$, which form the codebook. The encoding or the quantization process is denoted as $\widehat{\mathbf{y}}=\mathcal{Q}(\mathbf{y}, \mathbf{z})$. The distortion of a finite rate quantizer is defined as

$$
D=E_{\mathbf{x}}\left[D_{\mathrm{Q}}(\mathbf{y}, \widehat{\mathbf{y}} ; \mathbf{z})\right],
$$

where $D_{\mathrm{Q}}(\mathbf{y}, \widehat{\mathbf{y}} ; \mathbf{z})$ is a general non mean-squared distortion function between $\mathbf{y}$ and $\widehat{\mathbf{y}}$ that is parameterized by $\mathbf{z}$. It is further assumed that function $D_{\mathrm{Q}}$ has a continuous second order derivative (or Hessian matrix w.r.t. to $\mathbf{y}) \mathbf{W}_{\mathbf{z}}(\widehat{\mathbf{y}})$ with the $(i, j)^{\text {th }}$ element given by

$$
w_{i, j}=\left.\frac{1}{2} \cdot \frac{\partial^{2}}{\partial y_{i} \partial y_{j}}\right|_{\mathbf{y}=\widehat{\mathbf{y}}} D_{\mathrm{Q}}(\mathbf{y}, \widehat{\mathbf{y}} ; \mathbf{z}) .
$$

Under high resolution assumptions, the asymptotic distortion of a finite rate feedback system can be represented by the following form [9], which is similar to the Bennett's integral provided in [10]

$$
\begin{aligned}
D & =E_{\mathbf{y}, \mathbf{z}}\left[D_{\mathbf{Q}}(\mathbf{y}, Q(\mathbf{y}, \mathbf{z}) ; \mathbf{z})\right] \\
& =2^{-\frac{2 B}{k_{\mathrm{q}}}} \int_{\mathbb{Z}} \int_{\mathbb{Q}} I\left(\mathbf{y} ; \mathbf{z} ; \mathbb{E}_{\mathbf{z}}(\mathbf{y})\right) p(\mathbf{y}, \mathbf{z}) \lambda(\mathbf{y})^{-\frac{2}{k_{\mathrm{q}}}} d \mathbf{y} d \mathbf{z},(8)
\end{aligned}
$$

where $\mathbb{E}_{\mathbf{z}}(\mathbf{y})$ denotes the asymptotic projected Voronoi cell that contains $\mathbf{y}$ with side information $\mathbf{z}$ as $N$ approaches infinity. In equation $(8), \lambda(\mathbf{y})$ is a function representing the relative density of the codepoints, which is called point density, such that $\lambda(\mathbf{y}) d \mathbf{y}$ is approximately the fraction of quantization points in a small neighborhood of $\mathbf{y}$. Function $I(\mathbf{y} ; \mathbf{z} ; \mathbb{E})$ is the normalized moment of inertia profile that represents the asymptotic normalized distortion or the relative distortion of the quantizer $\mathcal{Q}$ at position $\mathbf{y}$ conditioned on side information $\mathbf{z}$ with Voronoi shape $\mathbb{E}$. Both $\lambda(\mathbf{y})$ and $I(\mathbf{y} ; \mathbf{z} ; \mathbb{E})$ are the key performance determining characteristics that can be used to analyze the effects of different system parameters, such as source distribution, distortion function, quantization rate etc., on the finite rate quantizer.

The normalized moment of inertia profile of an optimal quantizer is defined as the minimum moment of inertia of 
all admissible regions $\mathbb{E}_{\mathbf{z}}(\mathbf{y})$, i.e.

$$
I_{\mathrm{opt}}(\mathbf{y} ; \mathbf{z}) \triangleq \min _{\mathbb{E}_{\mathbf{z}}(\mathbf{y}) \in \mathcal{H}_{\mathrm{Q}}} I\left(\mathbf{y} ; \mathbf{z} ; \mathbb{E}_{\mathbf{z}}(\mathbf{y})\right)
$$

with $\mathcal{H}_{\mathrm{Q}}$ representing the class of all admissible polytopes that forms a lattice partition on the quantization space $\mathbb{Q}$. The optimal moment of inertia can be tightly lower bounded by

$$
I_{\text {opt }}(\mathbf{y} ; \mathbf{z}) \gtrsim \widetilde{I}_{\text {opt }}(\mathbf{y} ; \mathbf{z})=\frac{k_{\mathrm{q}}}{k_{\mathrm{q}}+2} \cdot\left(\frac{\left|\mathbf{W}_{\mathbf{z}}(\mathbf{y})\right|}{\kappa_{k_{\mathrm{q}}}^{2}}\right)^{1 / k_{\mathrm{q}}},
$$

where parameter $\kappa_{n}$ is the volume of a $n$-dimensional unit sphere given by

$$
\kappa_{n}=\frac{\pi^{n / 2}}{\Gamma(n / 2+1)} .
$$

The asymptotic distortion of an optimal finite rate quantizer can be lower bounded by the following form

$$
D\left(\mathcal{Q}_{\text {opt }}\right) \geq D_{\text {Low }, 1},
$$

where lower bound $D_{\text {Low, } 1}$ is given by

$$
D_{\text {Low }, 1}=2^{-\frac{2 B}{k_{\mathrm{q}}}} \cdot\left(\int_{\mathbb{Q}}\left(I_{\mathrm{opt}}^{\mathrm{w}}(\mathbf{y}) \cdot p(\mathbf{y})\right)^{1 /\left(1+\frac{2}{k_{\mathrm{q}}}\right)} d \mathbf{y}\right)^{1+\frac{2}{k_{\mathrm{q}}}},
$$

with the average moment of inertia profile $I_{\mathrm{opt}}^{\mathrm{w}}(\mathbf{y})$ given by

$$
I_{\mathrm{opt}}^{\mathrm{w}}(\mathbf{y})=\int_{\mathbb{Z}} I_{\mathrm{opt}}(\mathbf{y} ; \mathbf{z}) \cdot p(\mathbf{z} \mid \mathbf{y}) d \mathbf{z}
$$

When the sensitivity matrix $\mathbf{W}_{\mathbf{z}}(\mathbf{y})$ can be factored into the following form

$$
\mathbf{W}_{\mathbf{z}}(\mathbf{y})=f(\mathbf{z}) \cdot \mathbf{W}(\mathbf{y}),
$$

it can be shown that the asymptotic distortion lower bound $D_{\text {Low, } 1}$ is actually achievable

$$
D\left(\mathcal{Q}_{\text {opt }}\right)=D_{\text {Low }, 1} \text {. }
$$

By substituting the tight lower bound (10) of the moment of inertia profile into equations (13) and (14), we can obtain corresponding tight lower bound $\widetilde{I}_{\text {opt }}^{\mathrm{w}}(\mathbf{y})$ of the average moment of inertial profile, and the lower bound $\widetilde{D}_{\text {Low, } 1}$ of the asymptotic system distortion respectively ${ }^{1}$.

If the source variable (vector) $\mathbf{y}$ is further subject to $k_{\mathrm{c}}$ constraints given by the vector equation

$$
\mathbf{g}(\mathbf{y})=0 \text {, }
$$

the asymptotic distortion analysis is still valid with the following modifications. First, the actual degrees of freedom of the quantization variable reduce from $k_{\mathrm{q}}$ to $k_{\mathrm{q}}^{\prime}=k_{\mathrm{q}}-k_{\mathrm{c}}$. Second, the sensitivity matrix is replaced by its constrained version $\mathbf{W}_{\mathrm{c}, \mathbf{z}}(\mathbf{y})$ given by

$$
\mathbf{W}_{\mathrm{c}, \mathbf{z}}(\mathbf{y})=\mathbf{V}_{2}^{T} \cdot \mathbf{W}_{\mathbf{z}}(\mathbf{y}) \cdot \mathbf{V}_{2}
$$

where $\mathbf{V}_{2} \in \mathbb{R}^{k_{\mathrm{q}} \times k_{\mathrm{q}}^{\prime}}$ is an orthonormal matrix with its columns constituting an orthonormal basis for the orthogonal compliment of the range space $\mathcal{R}\left(\frac{\partial}{\partial \mathbf{y}} \mathbf{g}(\mathbf{y})\right)$. Lastly, the multi-dimensional integrations used in evaluating $D_{\text {Low, } 1}$ and $\widetilde{D}_{\text {Low, } 1}$ is performed in the constraint space $\mathbf{g}(\mathbf{y})=0$.

\footnotetext{
${ }^{1}$ This replacement can be extended to other variables and definitions. In the rest of this paper, we will directly use $\widetilde{a}$ to represent a quantity that is obtained by replacing $I_{\mathrm{opt}}$ with $\widetilde{I}_{\mathrm{opt}}$ if $a$ is a function of $I_{\mathrm{opt}}$, i.e. $a=a\left(I_{\mathrm{opt}}\right)$
}

\section{UNCORRELATED MISO BEAMFORMING SYSTEMS}

Although the analysis of finite rate quantized MISO beamforming system over i.i.d. Rayleigh fading channels has been investigated in several past works, we revisit this problem from a source coding perspective by formulating it into a general vector quantization problem and provide analysis based on the general framework.

The source variable is denoted as $\overline{\mathbf{v}}=\left[\mathbf{v}_{\mathrm{R}}^{T}, \mathbf{v}_{\mathrm{I}}^{T}\right]^{T}$ of dimension $2 t$ with $\mathbf{v}_{\mathrm{R}}$ and $\mathbf{v}_{\mathrm{I}}$ representing the real and imaginary parts of the (complex) channel directional vector $\mathbf{v}$, and the side information is denoted as $\alpha=\|\mathbf{h}\|^{2}$ of dimension $k_{\alpha}=1$. For vectors in the vicinity of $\widehat{\mathbf{v}}$ (with $\widehat{\mathbf{v}}_{R}$ and $\widehat{\mathbf{v}}_{\text {I }}$ representing its real and imaginary parts), source variable $\overline{\mathbf{v}}$ is constrained under the multi-dimensional real function $\mathbf{g}(\overline{\mathbf{v}})$ corresponding to definition (17), which is given by

$$
\mathbf{g}(\mathbf{v})=\left[\begin{array}{l}
\mathbf{v}_{\mathrm{R}}^{T} \mathbf{v}_{\mathrm{R}}+\mathbf{v}_{\mathrm{I}}^{T} \mathbf{v}_{\mathrm{I}}-1 \\
\mathbf{v}_{\mathrm{R}}^{T} \widehat{\mathbf{v}}_{\mathrm{I}}-\mathbf{v}_{\mathrm{I}}^{T} \widehat{\mathbf{v}}_{\mathrm{R}}
\end{array}\right]=0,
$$

with the first element representing the norm constraint and the second element the phase constraint. Function $\mathbf{g}(\mathbf{v})$ has size $k_{\mathrm{c}}=2$, which leads to the actual degrees of freedom of the quantization variable $\mathbf{v}$ to be $k_{\mathrm{q}}^{\prime}=2 t-2$. The instantaneous capacity loss due to effects of finite rate CSI quantization is taken to be the system distortion function $D_{\mathrm{Q}}(\mathbf{v}, \widehat{\mathbf{v}} ; \alpha)$, which has the following second order approximation

$$
\begin{aligned}
D_{\mathrm{Q}}(\mathbf{v}, \widehat{\mathbf{v}} ; \alpha) & =-\log _{2}\left(1-\frac{\rho \alpha}{1+\rho \alpha} \cdot\left(1-|\langle\mathbf{v}, \widehat{\mathbf{v}}\rangle|^{2}\right)\right) \\
& \approx \frac{\rho \alpha}{\ln 2(1+\rho \alpha)} \cdot \overline{\mathbf{v}}^{T}\left(I_{2 t}-\mathbf{\Omega}\right) \overline{\mathbf{v}},
\end{aligned}
$$

where matrix $\Omega \in \mathbb{R}^{2 k_{\mathrm{q}} \times 2 k_{\mathrm{q}}}$ is given by

$$
\boldsymbol{\Omega}=\left[\begin{array}{c|c}
\widehat{\mathbf{v}}_{\mathrm{R}} \widehat{\mathbf{v}}_{\mathrm{R}}^{T}+\widehat{\mathbf{v}}_{\mathrm{I}} \widehat{\mathbf{v}}_{\mathrm{I}}^{T} & \widehat{\mathbf{v}}_{\mathrm{R}} \widehat{\mathbf{v}}_{\mathrm{I}}^{T}-\widehat{\mathbf{v}}_{\mathrm{I}} \widehat{\mathbf{v}}_{\mathrm{R}}^{T} \\
\hline \widehat{\mathbf{v}}_{\mathrm{I}} \widehat{\mathbf{v}}_{\mathrm{R}}^{T}-\widehat{\mathbf{v}}_{\mathrm{R}} \widehat{\mathbf{v}}_{\mathrm{I}}^{T} & \widehat{\mathbf{v}}_{\mathrm{R}} \widehat{\mathbf{v}}_{\mathrm{R}}^{T}+\widehat{\mathbf{v}}_{\mathrm{I}} \widehat{\mathbf{v}}_{\mathrm{I}}^{T}
\end{array}\right] .
$$

After some manipulations, the constrained sensitivity matrix $\mathbf{W}_{\mathrm{c}, \alpha}(\widehat{\mathbf{v}})$ can be shown to be given by, from equation (18)

$$
\mathbf{W}_{\mathrm{c}, \alpha}(\widehat{\mathbf{v}})=\frac{\rho \alpha}{\ln 2 \cdot(1+\rho \alpha)} \cdot I_{2 t-2} .
$$

Substituting (22) into (10), the optimal moment of inertia profile can be obtained by the following form

$$
\begin{aligned}
I_{\mathrm{c}, \text { opt }}(\widehat{\mathbf{v}} ; \alpha) & =I_{\mathrm{c}, \text { opt }}(\alpha) \gtrsim \widetilde{I}_{\mathrm{c}, \mathrm{opt}}(\alpha) \\
& =\frac{\rho \alpha}{\ln 2 \cdot(1+\rho \alpha)} \cdot \frac{(t-1) \cdot \gamma_{t}^{-1 /(t-1)}}{t}
\end{aligned}
$$

where the parameter $\gamma_{t}$ is given by

$$
\gamma_{t}=\frac{\pi^{t-1}}{(t-1) !}
$$

When the elements of the channel response $\mathbf{h}$ are i.i.d. Gaussian distributed, the overall system distortion (or the capacity loss) can be obtained from (13) and has the following form

$$
\begin{aligned}
& \widetilde{D}\left(\mathcal{Q}_{\text {opt }}\right)=\widetilde{D}_{\text {c-Low }, 1} \\
& \quad=\frac{(t-1) \cdot\left({ }_{2} F_{0}(t+1,1 ; ;-\rho) \cdot \rho\right)}{\ln 2} \cdot 2^{-B /(t-1)},
\end{aligned}
$$

where ${ }_{2} F_{0}$ is the generalized hypergeometric function, and the optimal point density $\lambda^{*}(\mathbf{v})$ is a uniform distribution given by

$$
\lambda^{*}(\mathbf{v})=\gamma_{t}^{-1}, \quad \mathbf{v} \in\{\mathbf{v} \mid \mathbf{g}(\mathbf{v})=0\} .
$$




\section{Correlated Miso Beamforming Systems}

It is generally difficult to extend the analysis of quantized MISO systems from i.i.d. channels to correlated channels by using the statistical approximation approach adopted in [8] and other previous works. By viewing the problem using a source coding perspective, we provide in this section the distortion analysis of correlated MISO channels.

\section{A. Distortion lower bound $D_{c-L o w, 1}\left(\boldsymbol{\Sigma}_{h}\right)$}

When the elements of the MISO channel response $\mathbf{h}$ are correlated and have a complex Gaussian distribution, i.e. $\mathbf{h} \sim \mathcal{N}_{\mathrm{c}}\left(\mathbf{0}, \boldsymbol{\Sigma}_{\mathrm{h}}\right)$, by substituting the moment of inertia profile $I_{\mathrm{c} \text {, opt }}(\mathbf{v} ; \alpha)$ given by (23) into equation (13), the system asymptotic distortion lower bound $\widetilde{D}_{\text {c-Low, } 1}$ can be expressed in the following form,

$$
\begin{aligned}
& D_{\text {c-Low }, 1}\left(\boldsymbol{\Sigma}_{\mathrm{h}}\right) \gtrsim \widetilde{D}_{\text {c-Low }, 1}\left(\boldsymbol{\Sigma}_{\mathrm{h}}\right) \\
& \quad=\frac{(t-1) \gamma_{t}^{-t /(t-1)} \cdot \rho \cdot \beta_{1}\left(\rho, t, \boldsymbol{\Sigma}_{\mathrm{h}}\right)}{\ln 2 \cdot\left|\boldsymbol{\Sigma}_{\mathrm{h}}\right|} \cdot 2^{-B /(t-1)}
\end{aligned}
$$

where $\beta_{1}\left(\rho, t, \boldsymbol{\Sigma}_{\mathrm{h}}\right)$ is a constant coefficient which only depends on the antenna size $t$, channel correlation matrix $\boldsymbol{\Sigma}_{\mathrm{h}}$ and system $\operatorname{SNR} \rho$, and is given by

$$
\begin{aligned}
& \beta_{1}\left(\rho, t, \boldsymbol{\Sigma}_{\mathbf{h}}\right)=\left(\int _ { \mathbf { v } : \mathbf { g } ( \mathbf { v } ) = 0 } \left(\left(\mathbf{v}^{H} \mathbf{\Sigma}_{\mathbf{h}}^{-1} \mathbf{v}\right)^{-(t+1)}\right.\right. \\
& \left.\left.\times{ }_{2} F_{0}\left(t+1,1 ; ;-\frac{\rho}{\mathbf{v}^{H} \mathbf{\Sigma}_{\mathrm{h}}^{-1} \mathbf{v}}\right)\right)^{(t-1) / t} d \mathbf{v}\right)^{t /(t-1)}
\end{aligned}
$$

The optimal point density $\lambda^{*}(\mathbf{v})$ that achieves the minimal distortion is given by

$$
\begin{aligned}
\lambda^{*}(\mathbf{v}) & =\beta_{1}\left(\rho, t, \boldsymbol{\Sigma}_{\mathrm{h}}\right)^{-(t-1) / t} \cdot\left(\left(\mathbf{v}^{H} \boldsymbol{\Sigma}_{\mathrm{h}}^{-1} \mathbf{v}\right)^{-(t+1)}\right. \\
& \left.\times{ }_{2} F_{0}\left(t+1,1 ;-\frac{\rho}{\mathbf{v}^{H} \boldsymbol{\Sigma}_{\mathrm{h}}^{-1} \mathbf{v}}\right)\right)^{(t-1) / t} .
\end{aligned}
$$

All the derivations provided in this article are brief due to space limitations.

From the proposed distortion lower bound $\widetilde{D}_{\text {c-Low,1 }}\left(\boldsymbol{\Sigma}_{\mathrm{h}}\right)$, we can make the following observations:

1) The asymptotic distortion lower bound provided in equation (27) is a general format that is suitable for arbitrary channel correlations with covariance matrix $\boldsymbol{\Sigma}_{\mathrm{h}}$. The distortion lower bound of i.i.d. MISO channels given by equation (25) is a special case when the covariance matrix $\boldsymbol{\Sigma}_{\mathrm{h}}$ is equal to the identity matrix, i.e. $\boldsymbol{\Sigma}_{\mathrm{h}}=I_{t}$.

2) Since the sensitivity matrix $\mathbf{W}_{\mathrm{c}, \alpha}(\widehat{\mathbf{v}})$ given by (22) satisfies the factorable condition given by equation (15), the distortion lower bound $D_{\text {c-Low, } 1}$ is achievable and equal to the asymptotic distortion of the optimal quantizer, i.e.

$$
D_{\text {c-Low }, 1}=D_{\text {Q-opt }} \gtrsim \widetilde{D}_{\text {c-Low, } 1}=\widetilde{D}_{\text {Q-opt }} .
$$

3) It can be observed from equations (25) and (27) that the distortions of correlated and i.i.d. MISO channels have the same exponential decaying factor $2^{-B /(t-1)}$ with the feedback rate $B$. Furthermore, it can also be shown that the distortion lower bound $\widetilde{D}_{\text {c-Low,1 }}$ of correlated MISO channels is smaller than that of the i.i.d. MISO channels [9], i.e.

$$
0<\widetilde{D}_{\mathrm{c}-\text { Low }, 1}\left(\boldsymbol{\Sigma}_{\mathrm{h}}\right) \stackrel{a}{\leq} \widetilde{D}_{\mathrm{c}-\text { Low }, 1}\left(I_{t}\right)
$$

with equality in $(a)$ if and only if $\boldsymbol{\Sigma}_{\mathrm{h}}=I_{t}$. This means that i.i.d. channels are the worst channel to quantize in the sense of having the largest distortion or capacity loss.

\section{B. Analysis in High-SNR Regimes}

In high SNR regimes, the constraint sensitivity matrix $\mathbf{W}_{\mathrm{c}, \alpha}$ reduces to be

$$
\mathbf{W}_{\mathrm{c}, \alpha}^{\mathrm{H}-\mathrm{snr}}(\mathbf{v}, \alpha)=\lim _{\rho \rightarrow \infty} \frac{\rho \alpha}{\ln 2 \cdot(1+\rho \alpha)} \cdot I=\frac{I}{\ln 2},
$$

which is independent of $\mathbf{v}$, the side information information $\alpha$ as well as the SNR $\rho$. This means that 1) the encoder can discard the available side information $\alpha$ without any loss of system performance; 2) one single codebook is used for different system SNRs in high SNR regions. In this case, the moment of inertia profile $\widetilde{I}_{\mathrm{opt}}(\mathbf{v}, \alpha)$ and the average moment of inertia profile $\widetilde{I}_{\mathrm{opt}}^{\mathrm{w}}(\mathbf{v}, \alpha)$ also reduce to be constants independent of the location $\mathbf{v}$ as well as side information $\alpha$

$$
\widetilde{I}_{\mathrm{opt}}^{\mathrm{H}-\mathrm{snr}}=\widetilde{I}_{\mathrm{opt}}^{\mathrm{w}, \mathrm{H}-\mathrm{snr}}=\frac{(t-1) \cdot \gamma_{t}^{-1 /(t-1)}}{\ln 2 \cdot t} .
$$

By substituting (33) into the distortion lower bound given by (13), the system capacity loss of i.i.d. MISO channels in high SNR regime is given by

$$
\widetilde{D}_{\text {c-Low }, 1}^{\mathrm{H} \text {-snr }}\left(\boldsymbol{\Sigma}_{\mathrm{h}}=I_{t}\right)=\frac{t-1}{t} \cdot 2^{-B /(t-1)},
$$

which is consistent with the analysis obtained in [8] based on a statistical approach. For correlated MISO channels, the high-SNR asymptotic distortion lower bound $\widetilde{D}_{\text {c-Low.1 }}^{\mathrm{H} \text {-snr }}$ can be shown to have the following form, from equation (27)

$$
\begin{aligned}
\widetilde{D}_{\mathrm{c}-\text { Low }, 1}^{\mathrm{H} \text { snr }}\left(\boldsymbol{\Sigma}_{\mathrm{h}}\right) & =\frac{(t-1) \cdot\left(\prod_{i=1}^{t} \lambda_{\mathrm{h}, i}\right)^{1 /(t-1)}}{\ln 2 \cdot t} \cdot \\
& \left((t-1) \sum_{i=1}^{t} \frac{\left(\ln \lambda_{\mathrm{h}, i}\right) / \lambda_{\mathrm{h}, i}}{\prod_{k \neq i}\left(1-\lambda_{\mathrm{h}, k} / \lambda_{\mathrm{h}, i}\right)}\right)^{t /(t-1)} \cdot 2^{-B /(t-1)},
\end{aligned}
$$

where $\left.\lambda_{\mathrm{h}, i}\right|_{i=1} ^{t}$ are the eigen-values of matrix $\boldsymbol{\Sigma}_{\mathrm{h}}$.

\section{Mismatched AnAlysis OF MISO Systems With FINITE RATE FEEDBACK}

In the previous sections, we provided asymptotic analysis of MISO systems with optimal CSI quantizer, in the sense that the codebook or the encoding algorithm is designed to perfectly match the distortion function and the source distribution. However, imperfect codebook and suboptimal quantizer might be used in practical situations in order to reduce the design and encoding complexity or due to imperfect knowledge of the source distribution. For example the codebook designed for i.i.d channels could be employed in a correlated environment. We provide in this section a capacity loss analysis of the quantized MISO beamforming system when the quantizer is mismatched and suboptimal. The results further serve to demonstrate the usefulness and generality of the proposed framework. 


\section{A. Source Distribution Mismatch (or Point Density Mismatch)}

For the correlated MISO channels, the channel distribution depends on the covariance matrix $\Sigma_{\mathrm{h}}$, which needs to be estimated both at the transmitter and the receiver. A mismatch between the measured covariance matrix $\Sigma_{\mathrm{h}}^{\mathrm{m}}$ and the actual $\boldsymbol{\Sigma}_{\mathrm{h}}$ will cause system performance degradation.

Based on the mismatched covariance matrix $\Sigma_{\mathrm{h}}^{\mathrm{m}}$, a suboptimal codebook is generated with the mismatched point density given by, from equation (29),

$$
\begin{array}{r}
\lambda_{\text {mis }}(\mathbf{v})=\beta_{1}\left(\rho, t, \boldsymbol{\Sigma}_{\mathrm{h}}^{\mathrm{m}}\right)^{-(t-1) / t}\left(\left(\mathbf{v}^{H}\left(\boldsymbol{\Sigma}_{\mathrm{h}}^{\mathrm{m}}\right)^{-1} \mathbf{v}\right)^{-(t+1)}\right. \\
\left.{ }_{2} F_{0}\left(t+1,1 ;-\frac{\rho}{\mathbf{v}^{H}\left(\boldsymbol{\Sigma}_{\mathrm{h}}^{\mathrm{m}}\right)^{-1} \mathbf{v}}\right)\right)^{(t-1) / t} \cdot \quad
\end{array}
$$

By substituting the mismatched point density $\lambda_{\text {mis }}$ given by (36) into the distortion integral (8), the system distortion lower bound of the mismatched quantizer can be obtained

$$
\begin{aligned}
& \widetilde{D}_{\text {mis-P-Low } 1}=\left(\int_{\mathbf{x}: \mathbf{g}(\mathbf{x})=0} \widetilde{I}_{\mathrm{c}, \mathrm{opt}}^{\mathrm{w}}(\mathbf{x}) .\right. \\
&\left.p_{\mathbf{v}}(\mathbf{x}) \cdot \lambda_{\text {mis }}(\mathbf{x})^{-1 /(t-1)} d \mathbf{x}\right) \cdot 2^{-B /(t-1)} .
\end{aligned}
$$

As a special case, if the codebook designed for i.i.d. MISO channels is used for correlated MISO systems ${ }^{2}$, i.e. $\boldsymbol{\Sigma}_{\mathrm{h}}^{\mathrm{m}}=I_{t}$, the mismatched point density $\lambda_{\text {mis }}(\mathbf{v})$ is uniform and the asymptotic distortion of the mismatched quantizer can be obtained by the following analytical closed form expression after some manipulations

$$
\widetilde{D}_{\text {mis-P-Low, } 1}\left(\boldsymbol{\Sigma}_{\mathrm{h}}\right)=\frac{(t-1) \cdot \beta_{5}\left(\rho, \boldsymbol{\Sigma}_{\mathrm{h}}\right)}{\ln 2 \cdot t} \cdot 2^{-B /(t-1)},
$$

where the constant coefficient $\beta_{5}\left(\rho, \boldsymbol{\Sigma}_{\mathrm{h}}\right)$ is given by

$$
\begin{aligned}
\beta_{5}\left(\rho, \boldsymbol{\Sigma}_{\mathrm{h}}\right)= & 1+\sum_{i=1}^{t}\left(\rho \lambda_{\mathrm{h}, i} \prod_{j \neq i}\left(1-\frac{\lambda_{\mathrm{h}, j}}{\lambda_{\mathrm{h}, i}}\right)\right)^{-1} \\
& \cdot \exp \left(\frac{1}{\rho \lambda_{\mathrm{h}, i}}\right) \cdot E_{\mathrm{i}}\left(\frac{-1}{\rho \lambda_{\mathrm{h}, i}}\right)
\end{aligned}
$$

where $E_{\mathrm{i}}(\cdot)$ is the exponential integral function.

\section{B. Comparison With Other Quantizers}

In order to understand how the mismatched channel covariance matrix $\left(\Sigma_{\mathrm{h}}^{\mathrm{m}}=I_{t}\right)$ affects the MISO system performance, a distortion comparison between optimal and mismatched quantizers under both correlated and i.i.d. fading channels is formed. By utilizing the concavity property of function $\beta_{5}\left(\rho, \boldsymbol{\Sigma}_{\mathrm{h}}\right)$ w.r.t. matrix $\boldsymbol{\Sigma}_{\mathrm{h}}$, it can be proved that $\widetilde{D}_{\text {mis-P-Low, } 1}\left(\boldsymbol{\Sigma}_{\mathrm{h}}\right)$ satisfies the following inequality

$$
\widetilde{D}_{\text {c-Low }, 1}\left(\boldsymbol{\Sigma}_{\mathrm{h}}\right) \leq \widetilde{D}_{\text {mis-P-Low, } 1}\left(\boldsymbol{\Sigma}_{\mathrm{h}}\right) \leq \widetilde{D}_{\text {c-Low }, 1}\left(I_{t}\right) .
$$

Moreover, it can also be shown that the mismatched system distortion $\widetilde{D}_{\text {mis-P-Low, } 1}\left(\boldsymbol{\Sigma}_{\mathrm{h}}\right)$ converges to the distortion of i.i.d.

\footnotetext{
${ }^{2}$ This can be also viewed as the case where the channel covariance matrix is completely unavailable at both the transmitter and the receiver.
}

MISO channels with optimal quantizers in high-SNR regimes, which is given by

$$
\left.\widetilde{D}_{\text {mis-P-Low, } 1}\left(\boldsymbol{\Sigma}_{\mathrm{h}}\right)\right|_{\rho \rightarrow \infty}=\widetilde{D}_{\text {c-Low }, 1}^{\mathrm{H}-\mathrm{snr}}\left(I_{t}\right)=\frac{t-1}{\ln 2 \cdot t} \cdot 2^{-B /(t-1)} \text {. }
$$

This means that: 1) Even with mismatched quantizers that use codebooks generated from i.i.d. channels samples, the system asymptotic distortion (or capacity loss) of correlated MISO channels is still less than that of the i.i.d. channels; 2) The performance of the mismatched quantizer is dominated by its suboptimal codebook, and hence does not depend on the channel correlations in high SNR regimes.

\section{NumericAl AND Simulation Results}

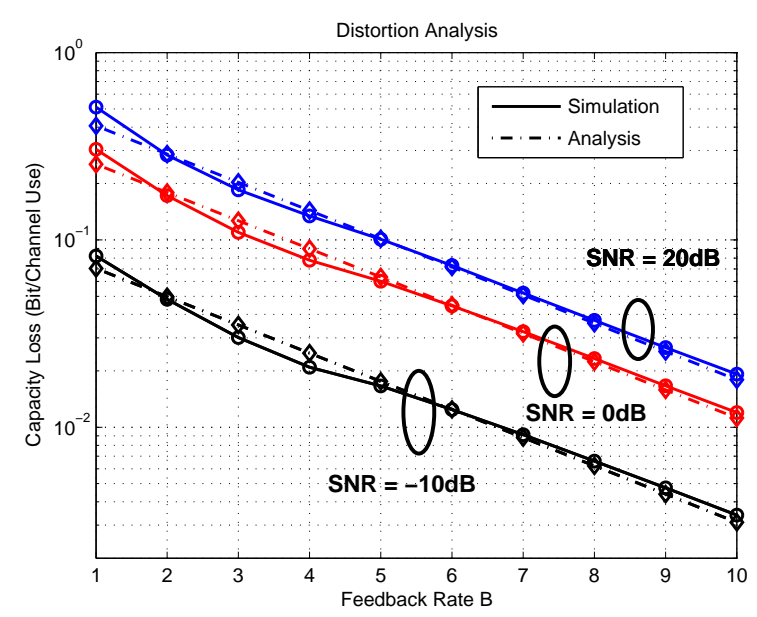

Fig. 1. Capacity loss of a $3 \times 1$ MISO system with different CSI feedback rate $B=1,2, \cdots, 10$ bits per channel update

We plot in Fig. 1 the system capacity loss due to the finiterate quantization of the CSI versus the feedback rate $B$ for a $3 \times 1$ MISO system over correlated fading channels. The spatially correlated channel is simulated by the correlation model in [11]: A linear antenna array with antenna spacing of half wavelength, uniform angular-spread in $\left[-30^{\circ}, 30^{\circ}\right]$ and angle of arrival $\phi=0^{\circ}$. Both the simulation results with codebook generated by the inner product criterion proposed in [8] and the analytical evaluation of distortion lower bound $\widetilde{D}_{\text {c-Low, } 1}$ given by $(27)$ are shown in the plot, demonstrating the accuracy of the proposed asymptotic distortion analysis provided in Section $\mathrm{V}$.

In order to see the effects of channel correlation on CSI quantization in a MISO system, we show in Fig. 2 the curves of capacity loss versus quantization rate (both simulation and analytical lower bound) of the same MISO system under different channel correlations with adjacent antenna spacing $D / \lambda=0.2,0.3,0.5,2.0$ and $\operatorname{SNR} \rho=20 \mathrm{~dB}$. As a comparison to uncorrelated MISO channels, we also show in Fig. 3 the distortion ratio of correlated MISO channels over i.i.d. fading channels with quantization rate $B=10$ bits, and under different channel correlations. It can be observed from the plot that the system distortion of correlated MISO channels is strictly less than that of the i.i.d. channels and the analytical result agree well with the actual simulation results. 


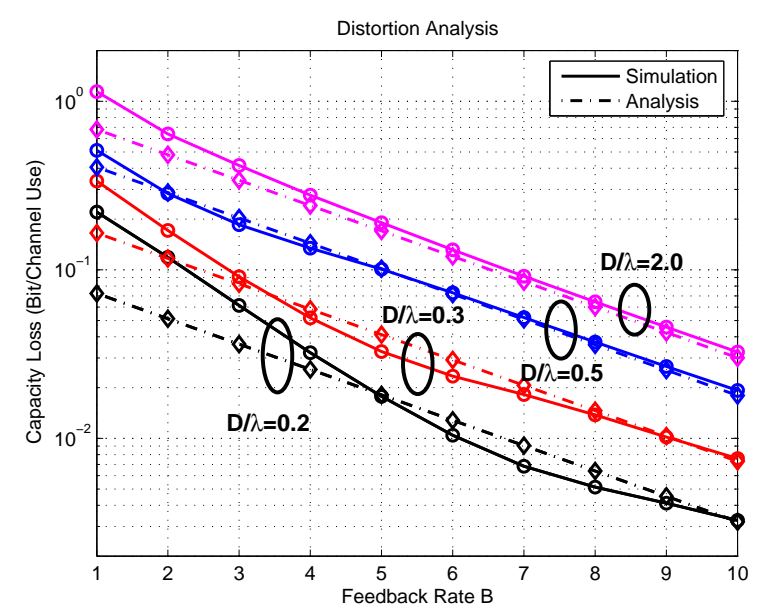

Fig. 2. Capacity loss versus CSI quantization rate of a $3 \times 1$ MISO system over different correlated fading channels of $D / \lambda=0.2,0.3,0.5,2.0$

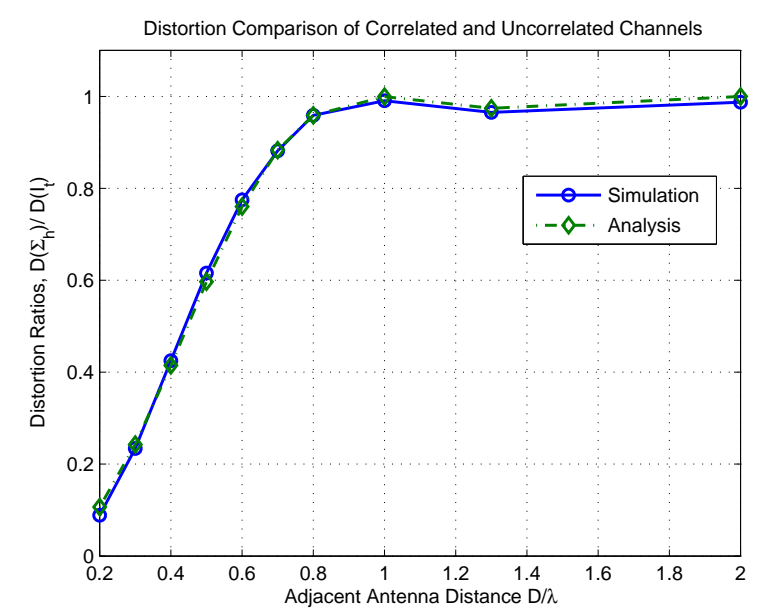

Fig. 3. Distortion comparison of correlated and uncorrelated MISO channels

We demonstrate in Fig. 4 the system capacity loss of the mismatched quantizer with codebook designed for a $3 \times 1$ i.i.d. MISO channel but used in a correlated fading environment with SNR $\rho=20 d B$. For comparison purpose, the distortion of the optimal quantizer over correlated MISO channel with $D / \lambda=0.3$ is also included in the plot. It can be observed that the capacity loss of the mismatched quantizer is worse than that of the optimal quantizers with optimal designed codebooks. Furthermore, it does not depend on the channel correlation in high SNR regimes and converges to that of i.i.d. channels.

\section{CONCLUSION}

In this paper, we have considered the analysis of transmit beamforming methods in multiple antenna systems with finite rate feedback. By employing a general framework which is based on a source coding perspective, the problem is formulated as a general vector quantization problem with encoder side information, constrained quantization space and non-mean-square distortion functions. By utilizing the highresolution distortion analysis of the generalized quantizer, we

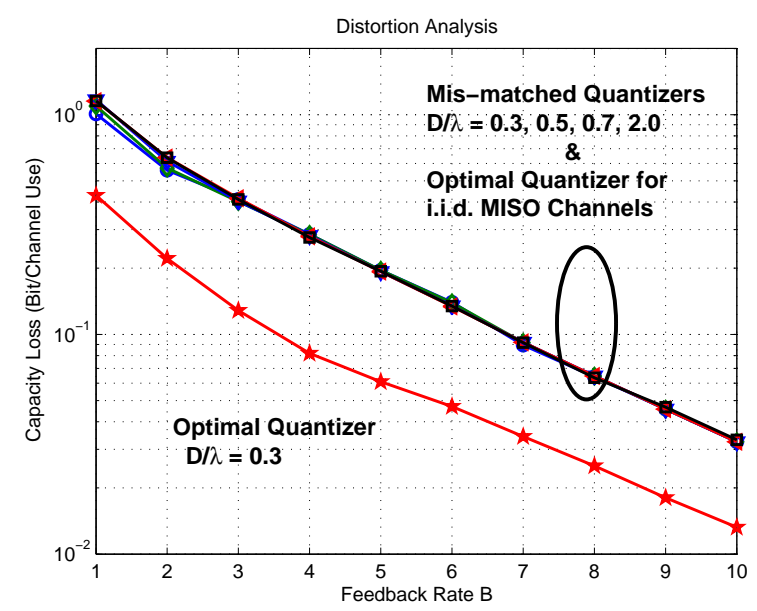

Fig. 4. Capacity loss of mismatched MISO quantizers with codebook designed for i.i.d. channels used in correlated fading environment

investigate the specific problem of MISO beamforming for spatially correlated channels. We obtain a tight lower bound on the capacity loss of the finite rate quantized MISO system, which is a generalization of existing results available on i.i.d. channels. The bound provides insight into the exact nature of dependence of the quantization loss on the channel correlation matrix, which indicates that the loss is less than that of the i.i.d. channels but with the same exponential decaying factor w.r.t. the feedback rate. The generality of the framework is further demonstrated by considering its application to the analysis of suboptimal mismatched channel quantizers, i.e. quantizers designed with an incorrect channel covariance matrix. Finally, numerical and simulation results of the finite rate quantized MISO beamforming are presented that confirm the accuracy of the obtained analytical results.

\section{REFERENCES}

[1] A. Narula, M. J. Lopez, M. D. Trott, and G. W. Wornell, "Efficient use of side information in multiple-antenna data transmission over fading channels," IEEE Journal on Selected Areas in Communications, vol. 16, pp. 1423-1436, Oct. 1998.

[2] A. Gersho and R. M. Gray, Vector Quantization and Signal Compression, Kluwer Academic Publishers, Massachusetts, 1992.

[3] K. K. Mukkavilli, A. Sabharwal, E.Erkip, and B.Aazhang, "On beamforming with finite rate feedback in multiple-antenna systems," IEEE Trans. On Information Theory, vol. 49, no. 10, pp. 2562-2579, Oct. 2003.

[4] D. J. Love, R. W. Heath, Jr., and T. Strohmer, "Grassmannian beamforming for multiple-input multiple-output wireless systems," IEEE Trans. on Information Theory, vol. 49, pp. 2735-2747, Oct. 2003.

[5] D. J. Love and R. W. Heath Jr., "Equal gain transmission in multipleinput multiple-output wireless systems," IEEE Trans. on Communications, vol. 51, no. 7, pp. 1102-1110, 2003.

[6] C. R. Murthy and B. D. Rao, "A vector quantization based approach for equal gain transmission," in Proc. Globecom, St. Louis, MO.

[7] P. Xia and G. B. Giannakis, "Design and analysis of transmitbeamforming based on limited-rate feedback," IEEE Trans. on Signal Processing, 2005 (to appear).

[8] June Chul Roh, Multiple-Antenna Communication with Finite Rate Feedback, Ph.D. thesis, Univ. of California, San Diego, 2005.

[9] J. Zheng and B. D. Rao, "Analysis of multiple antenna systems with finite rate feedback using high resolution quantization theory," IEEE Trans. on Signal Processing, in preparation.

[10] W. R. Bennett, "Spectra of quantized signals," Bell System Technical Journal, vol. 27, pp. 446-472, Jul 1948.

[11] J. Salz and J. H. Winters, "Effect of fading correlation on adaptive arrays in digital mobile radio," IEEE Trans. on Vehicular Technology, vol. 43, pp. 1049-1057, Nov. 1994. 